\title{
STUDY ON THE OPTIMIZATION OF VMS LOCATION BASED ON DRIVERS' GUIDANCE COMPLIANCE BEHAVIORS
}

\author{
Shiquan Zhong ${ }^{1}$, Lizhen Zhou ${ }^{2}$, Shoufeng $\mathrm{Ma}^{3}$, Xuelian Wang ${ }^{4}$, Ning $\mathrm{Jia}^{5}$ \\ 1,2,3,5College of Management and Economics, Tianjin University, Tianjin, China \\ ${ }^{4}$ School of Management, Hebei University of Technology, Tianjin, China
}

Submitted 22 April 2012; accepted 25 October 2012

\begin{abstract}
Traffic guidance compliance behavior is influenced by many factors. The present study investigates the effect of Variable Message Sign (VMS) location on guidance compliance behaviors of drivers. Based on the State, Operator, and Result (SOAR) cognitive architecture, a SOAR agent framework of drivers' traffic guidance compliance behavior is developed. The formation mechanism and the changes in the law of traffic guidance compliance behaviors of drivers, as well as the key factors of VMS location that affect drivers' compliance behaviors are studied. These factors include the visual perception of drivers, memory representation, decision cycle, and learning mechanisms. Finally, traffic guidance compliance behavior based on the SOAR cognitive architecture is simulated multiple times to verify the effectiveness of different VMS locations. The simulation results show that setting the VMS a bit further away from the downstream intersection achieves better guidance effect.
\end{abstract}

Keywords: traffic guidance, compliance behavior, VMS location, SOAR, multi-agent.

Reference to this paper should be made as follows: Zhong, S.; Zhou, L.; Ma, S.; Wang, X.; Jia, N. 2014. Study on the optimization of VMS location based on drivers' guidance compliance behaviors, Transport 29(2): 154-164.

http://dx.doi.org/10.3846/16484142.2014.929534

\begin{abstract}
Abbreviations
C: $\quad$ current vehicle;

$\mathrm{F}$ : front vehicle of $\mathrm{C}$ in the same lane;

LB: $\quad$ back vehicle of $\mathrm{C}$ in left adjacent lane;

LF: $\quad$ front vehicle of $\mathrm{C}$ in left adjacent lane;

LTM: long-term memory;

RB: back vehicle of $\mathrm{C}$ in right adjacent lane;

RF: front vehicle of $\mathrm{C}$ in right adjacent lane;

SOAR: state, operator and result;

STCA: symmetric two-lane cellular automata;

VMS: variable message sign;

WM: working memory.
\end{abstract}

\section{Sets}

$L 1$ : set of states in which $v_{L B}(t+1) \leq v_{L B}(t)$, $v_{L F}(t+1) \geq v_{L F}(t)$;

$L 2$ : set of states in which $v_{L B}(t+1)=v_{L B}(t)+1$, $v_{L F}(t+1)=v_{L F}(t)$;

L3: set of states in which $v_{L B}(t+1)=v_{L B}(t)$, $v_{L F}(t+1)=v_{L F}(t)-1$

L4: set of states in which $v_{L B}(t+1)=v_{L B}(t)+1$, $v_{L F}(t+1)=v_{L F}(t)-1$;
$R 1$ : set of states in which $v_{R B}(t+1) \leq v_{R B}(t)$, $v_{R F}(t+1) \geq v_{R F}(t)$;

$R 2$ : set of states in which $v_{R B}(t+1)=v_{R B}(t)+1$, $v_{R F}(t+1)=v_{R F}(t)$;

R3: set of states in which $v_{R B}(t+1)=v_{R B}(t)$, $v_{R F}(t+1)=v_{R F}(t)-1$;

R4: set of states in which $v_{R B}(t+1)=v_{R B}(t)+1$, $v_{R F}(t+1)=v_{R F}(t)-1$;

$S_{C}(t+1)$ : set of the possible values of $v_{C}(t+1)$.

\section{Variables}

$d\left(s_{k}, s_{e}\right):$ the distance between intermediate state $s_{k}$ and goal state $s_{e}$;

$d_{s}(s): \quad$ the sensed congestion degree under state $s$;

$d_{e}(s): \quad$ the number of destinations under state $s$;

$l_{C}$ :

$m_{o}(s)$ : the temper level of drivers under state $s$;

$n s_{i}: \quad$ the decision times between the current cycle and the cycle which generates rule $i$;

$n u_{i}: \quad$ the utilization times of rule $i$;

$N_{l}: \quad$ number of lane;

$N_{s}(l): \quad$ number of cells within the visual range of lane $l$; 
$N_{v}: \quad$ level number of the speed;

$o(s): \quad$ the applied operator under state $s$;

$p(t): \quad$ the congestion degree at time $t$;

$p_{c}: \quad$ the utilization ratio of procedural rules;

$P_{c}: \quad$ the critical utilization ratio of procedural rules;

$p_{q}: \quad$ the utilization ratio of episodic rules;

$P_{Q}^{L}: \quad$ the critical utilization lower bound;

$P_{Q}^{U}: \quad$ the critical utilization upper bound;

$p_{r}\left(s_{e}\right): \quad$ the total preference value under goal state $s_{e}$;

$p_{X}(t): \quad$ the location of vehicle $X$ at time $t$;

$r(s): \quad$ the transition path that involves state $s$;

$|r(s)|: \quad$ the number of states involved in $r(s)$;

$T\left(s_{e}\right): \quad$ the real driving time with goal state $s_{e}$;

$\bar{T}(v, l): \quad$ the mean driving time on road $l$ when the guidance information is $v$;

$T_{d}(t, l)$ : the mean driving time on road $l$ at time $t$;

$T_{r}(l): \quad$ the reference driving time on $\operatorname{road} l$;

$T_{v}[v(l)]:$ the effect of the shown congestion information $v$ on the driving time on road $l$;

$T_{e}\left(s_{e}\right): \quad$ the expected driving time with goal state $s_{e}$;

$v_{\max }: \quad$ maximum road speed;

$v_{X}(t)$ : the speed of vehicle $X$ at time $t$;

$\omega(l, c): \quad$ the weight of cell $c$ in lane $l$ to the congestion perceived by drivers;

$\lambda\left[d\left(s_{k}, s_{e}\right), r\left(s_{k}\right)\right]:$ the weighting of $p_{r}\left(s_{e}\right)$ for allocation to $o\left(s_{k}\right)$.

\section{Parameters}

$\alpha: \quad \quad$ used in the calculation of $p_{r}\left(s_{e}\right)$;

$\delta$ : $\quad$ a random number in the interval

$[-0.2,0.2]$;

$\eta_{z}$ : the threshold value of updating rules;

$\tau(s): \quad$ the threshold value of selecting operators;

$h(l, c)$ : $\quad 0$ if the cell $c$ of lane $l$ is occupied, and 1 otherwise;

$c_{c}^{1}, c_{c}^{2}, \beta_{c}$ : constants used in the calculation of $p_{c}$;

$c_{q}^{1}, c_{q}^{2}, \beta_{q}$ : constants used in the calculation of $p_{q}$;

$f_{1}, f_{2}, f_{3}, f_{4}$ : constants used in the calculation of $d\left(s_{k}, s_{e}\right)$.

\section{Introduction}

Traffic guidance compliance behavior is a basic problem, representing the perception of the guidance information of drivers, the degree of trust in the information, and the variation patterns in the degree of driver trust. Compliance rate is the external manifestation of drivers' guidance compliance behaviors; a high compliance rate is the foundation of an effective traffic guidance system (Wardman et al. 1997).
Two kinds of rate processing methods are employed in traditional theoretical guidance approaches and traditional system design. The first method presupposes the compliance rate, which is generally assumed to be $100 \%$ (Thakuriah, Sen 1996; Wang et al. 2006), or set as a constant between $0 \%$ and 100\% (Deflorio 2003; Yin, Yang 2003). The second one uses random utility theory and user equilibrium theory as bases instead of the compliance rate; the multinomial probability model (Mahmassani, Liu 1999; Jou et al. 2005), the theoretical model based on generalized extreme value (Bekhor, Prashker 2008), and the stochastic user equilibrium model (Daganzo, Sheffi 1977; Watling 2006), for examples. Regardless of the method, the guidance compliance rate is regarded as a static value. In practice, however, this rate is variable and changes with better understanding of the guidance system after its implementation. Whether drivers comply with guidance information or not, expected travel time and costs, perception of guidance information, familiarity with the road network, attitude toward risk, as well as the guidance information release mode and display mode are taken into consideration. All the elements and processes involved in the traffic guidance compliance behaviors should not be considered as absolute rationality. Jou et al. (2005) proved the bounded rationality of drivers' decisionmaking behavior. Theoretically, therefore, it is extremely difficult to describe driver's perception of guidance information, familiarity degree with the road network, and attitude toward risk, as well as the VMS location and the release mode of guidance information when traditional methods are adopted. Many researchers found that the actual compliance rate is different from the expected one. Many studies also analyzed the reasons of driver's low compliance rate. Drivers believe that:

- guidance information is unnecessary because they are familiar with the network;

- they do not notice, understand, or trust the guidance information;

- the information is received too late as the drivers have already chosen a path;

- the legibility distance of VMS is too short to read the guidance information, thereby causing them to miss the information (Bonsall, Joint 1991; Cummings 1994; Swann et al. 1995; Tarry, Graham 1995; Erke et al. 2007).

Thus, more detailed simulations of drivers' traffic guidance compliance behaviors are needed if guidance systems are expected to work effectively no matter in terms of theoretical analyses, practical investigation, or conclusions from scholars. Moreover, integrating multidisciplinary knowledge based on the multi-agent framework platform to study the traffic guidance compliance behavior has been receiving increasing attention. In the present study, SOAR cognitive architecture is introduced into the multi-agent framework, which is adopted as the platform to investigate the effects of VMS location on drivers' guidance compliance behaviors. 


\section{SOAR Cognitive Architecture}

SOAR is a general intelligent architecture developed by Laird et al. (1987). It is a cognitive architecture with a wide range of applications, and mainly focuses on knowledge, thinking, intelligence, and memory. SOAR is constructed with the assumption that all goal-oriented behaviors can be thought of as choosing an operator from a state. A state is a representation of the current problem-solving situation; an operator transforms a state (changes the representation) and produces a new state; and a goal is a desired outcome of the problemsolving activity. As SOAR runs, it is continually trying to apply the current operator and select the next operator (a state can have only a single operator at a time), until the goal is achieved.

As shown in Fig. 1 (Marinier et al. 2009), SOAR interacts with the environment through the perception and action interfaces. The environment is mapped into

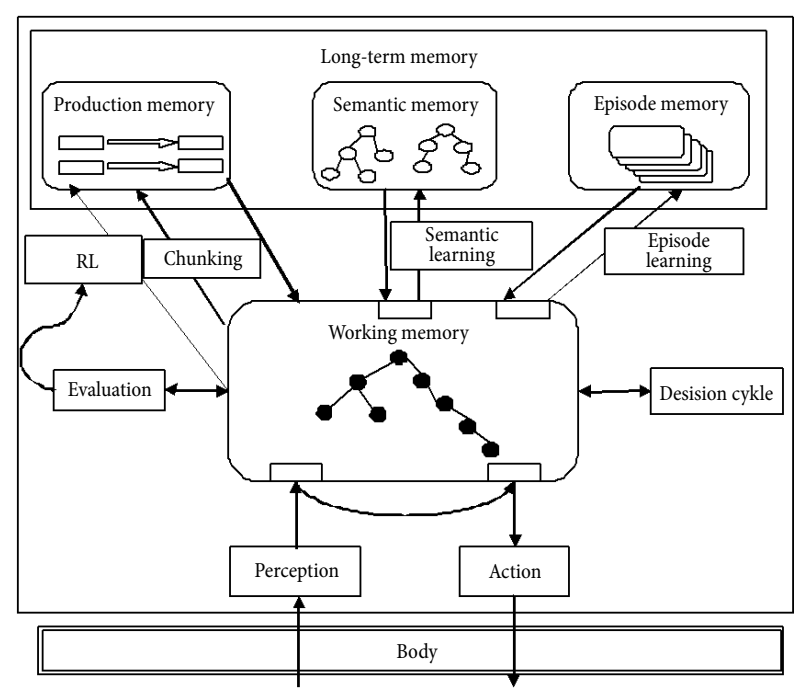

Fig. 1. SOAR architecture the WM through perception, and then the inner representations are returned to external environment and actions are directed to act on the environment through the action interface. SOAR has two kinds of memories with different forms of representation: the WM describes the current problem-solving situation, whereas the LTM stores long-term knowledge. In SOAR, the current situation, including data from the sensors, the results of intermediate inferences, active goals, and active operators, is held in the WM, which is represented as a hierarchical graph of states or goals. LTM contains production memory, semantic memory, and episodic memory. SOAR chooses and applies operators through a decisionmaking cycle, which is a fixed processing mechanism. Along with the decision-making cycle, SOAR has four different types of learning mechanisms, namely, reinforcement learning, chunking, episodic learning, and semantic learning. For a more detailed description of the modules and loops of SOAR, refer to Rosenbloom et al. (1987).

\section{SOAR Agent Framework of Traffic Guidance Compliance Behaviors}

The SOAR agent framework of traffic guidance and compliance behaviors of drivers is shown in Fig. 2 . SOAR agent perceives external traffic congestion, the state of the adjacent vehicle, the control information and guidance information as the input. After the input is incorporated into the $\mathrm{WM}$, the decision-making cycle, which includes input, state elaboration, proposing a candidate operator, selecting and applying an operator, is completed with support from the procedural and the episodic memory. Although many aspects are involved, only the key factors, which affect the effect of VMS location on traffic guidance compliance behaviors are investigated, including memory presentation, perception rules, decision-making cycle, and learning mechanisms.

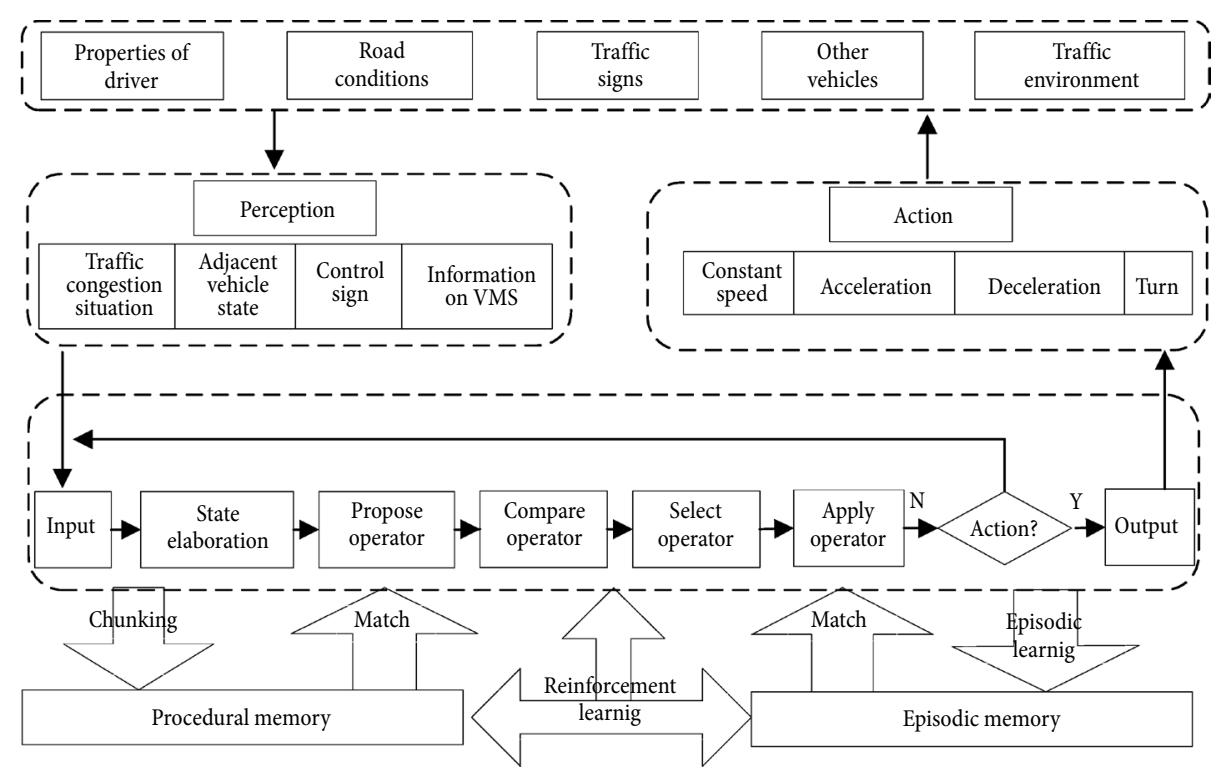

Fig. 2. SOAR agent framework of traffic guidance and compliance behaviors 


\section{Visual Perception of Drivers}

While driving, a driver perceives the external traffic environment through visual perception. In the scenario of traffic guidance compliance behavior in the present study, the adjacent vehicle state, traffic congestion within sight, and guidance information on a VMS are considered to be perceived visually.

\subsection{Adjacent Vehicle States}

The STCA model, proposed by Chowdhury et al. (1997), defines the lane changing rules of two-lane model:

- when the current vehicle accelerates or keeps limited speed, the front vehicle hinders it from maintaining its state;

- the distance between the current vehicle and the front vehicle on the adjacent lane satisfies the requirements for acceleration or maintaining limited speed;

- the distances between the current vehicle and the front vehicle, back vehicle on the adjacent lane are both greater than the safe distance. As three directions of VMS downstream roads, involving turning left, moving forward and turning right, have their own dedicated lanes for turning, five perception points are adopted to describe the adjacent vehicles (Fig. 3).

As shown in Fig. 3, the unfilled squares represent lane cells, the green-filled square denoted by $C$ represents the current vehicle, and the red-filled squares denoted by LB, LF, F, RB and RF represent the back vehicle on the left adjacent lane, the front vehicle on the left adjacent lane, the front vehicle on the same lane, the back vehicle on the right adjacent lane, and the front vehicle on the right adjacent lane, respectively. Suppose that the length of the cells is $l_{c}$, the maximum road speed is $v_{\max }$ (which is divided into $N_{v}$ intervals, $N_{v}=v_{\max } / l_{c}$ ), and the level number of the speed of each perception point is also $N_{v}$. Then, the actual speed range of level $k$ is $\left[(k-1) l_{c}, k l_{c}\right], k=1,2, \ldots, N_{v}$.

\subsection{Effects of the State of Adjacent Vehicles on Different Drivers' Lane-Changing Behaviors}

The goal of drivers in the STCA model is to seek the maximum speed, that is, if the front vehicle prevents the current vehicle from accelerating or maintaining the maximum speed, and the adjacent lane provides the opportunity to accomplish the goal, then drivers will turn to adjacent lane to avoid decelerating. This assumption is not true for the lane-changing goal depicted in the traffic guidance and compliance scenario, where the vehicles are all close to the intersection, drivers will change lanes to choose the best downstream road with the guidance from VMS. Once safety is secured, all lane-changing behaviors are manifested. In Fig. 4, the letters in the a)

\begin{tabular}{|c|c|c|c|c|}
\hline $\mathrm{a} 2$ & & & b3 & \\
\hline \multirow[t]{2}{*}{$\mathrm{d} 2$} & & $c 4$ & & \\
\hline & $\overline{\mathrm{e} 2}$ & & & f3 \\
\hline
\end{tabular}

$\mathrm{V} \longrightarrow$

b)

\begin{tabular}{|c|c|c|c|c|c|}
\hline a2 & & & c3 & b3 & \\
\hline \hline $\mathrm{d} 2$ & & & & & \\
\hline & $\mathrm{e} 2$ & & & & $\mathrm{f} 3$ \\
\hline
\end{tabular}

Fig. 4. Sketch maps of lane-changing in the guidance compliance scenario from $t$ to $t+1$

squares identify the vehicles, and the numbers represent the speeds of the vehicles.

As shown in Fig. 4, vehicle $\mathrm{C}$ moves forward at a speed of 4 . As no vehicle is positioned in front of it, $\mathrm{C}$ will not change lanes according to the STCA model. Moreover, even if $C$ wants to change lanes, a collision will happen between $\mathrm{C}$ and $\mathrm{B}$ if $\mathrm{C}$ accelerates or maintains its current speed. Hence, $\mathrm{C}$ cannot change lanes safely. Nevertheless, $\mathrm{C}$ can change lanes by decelerating, according to the lanechanging rules of traffic guidance compliance behaviors. Fig. 4 shows that when $C$ takes the left lane at a speed of 3 , it will not collide with B. This kind of lane-changing behavior is allowed in this guidance scenario. Suppose that the speeds of vehicles C, LB, LF, F, RB and RF are $v_{C}(t), v_{L B}(t), v_{L F}(t), v_{F}(t), v_{R B}(t)$ and $v_{R F}(t)$, and the locations of the vehicles are $p_{C}(t), p_{L B}(t), p_{L F}(t)$, $p_{F}(t), p_{R B}(t)$ and $p_{R F}(t)$. Take turning left as an example, the conditions required for lane changing without collision are $v_{C}(t+1)-v_{L B}(t+1)>p_{L B}(t)-p_{C}(t)$ and $v_{L F}(t+1)-v_{C}(t+1)>p_{C}(t)-p_{L F}(t)$. In the guidance scenario, the driver can accelerate or decelerate to satisfy theselane-changing conditions, that is, $v_{C}(t+1) \in S_{C}(t+1)$ and $S_{C}(t+1)=\left\{v_{C}(t)-1, v_{C}(t), v_{C}(t)+1\right\}$. The states at which drivers can change lanes while considering the speed of the other vehicles are as follows:

L1: $v_{L B}(t+1) \leq v_{L B}(t), v_{L F}(t+1) \geq v_{L F}(t)$.

In such a state, when vehicle $L B$ decelerates or maintains constant speed while LF accelerates or maintains constant speed, the condition for lanechanging $\forall v_{C}(t+1) \in S_{C}(t+1)$ should satisfy $p_{L B}(t)+v_{L B}(t)-p_{c}(t)<v_{C}(t+1)<v_{L F}(t)+p_{L F}(t)-p_{c}(t)$. L2: $v_{L B}(t+1)=v_{L B}(t)+1, v_{L F}(t+1)=v_{L F}(t)$.

In this scenario, when vehicle $L B$ accelerates while LF maintains constant speed, the condition for lane-changing $\forall v_{C}(t+1) \in S_{C}(t+1)$ should satisfy $p_{L B}(t)+v_{L B}(t)+1-p_{c}(t)<v_{C}(t+1)<v_{L F}(t)+p_{L F}(t)-p_{c}(t)$.

\begin{tabular}{|l|l|l|l|l|l|l|l|l|l|}
\hline & & $\mathrm{LB}$ & $\rightarrow$ & & $\mathrm{LF}$ & & $\rightarrow$ & & \\
\hline & & & $\rightarrow$ & $\mathrm{C}$ & & $\mathrm{F}$ & $\rightarrow$ & & \\
\hline & $\mathrm{RB}$ & & $\rightarrow$ & & & & $\rightarrow$ & $\mathrm{RF}$ & \\
\hline
\end{tabular}

Fig. 3. Locations of adjacent vehicles 
L3: $v_{L B}(t+1)=v_{L B}(t), v_{L F}(t+1)=v_{L F}(t)-1$. In this instance, when vehicle LB maintains constant speed while LF decelerates, the condition for lane-changing $\forall v_{C}(t+1) \in S_{C}(t+1)$ should satisfy $p_{L B}(t)+v_{L B}(t)-p_{c}(t)+1<v_{C}(t+1)<v_{L F}(t)+p_{L F}(t)-p_{c}(t)-1$. L4: $v_{L B}(t+1)=v_{L B}(t)+1, v_{L F}(t+1)=v_{L F}(t)-1$. When vehicle $L B$ accelerates while $L F$ decelerates, the condition for lane-changing $\forall v_{C}(t+1) \in S_{C}(t+1)$ should satisfy $p_{L B}(t)+v_{L B}(t)-p_{c}(t)+1<v_{C}(t+1)<v_{L F}(t)+p_{L F}(t)-p_{c}(t)-1$.

The agents of drivers in SOAR are divided according to their characteristics, that is, conservative one, general one and risk-based one. In states $L 1, L 2, L 3$ and $L 4$ (the corresponding right-lane-changing conditions are $R 1, R 2, R 3$ and $R 4$ ), the lane-changing conditions of different types of drivers are as follows: the conservative driver should satisfy $L 1$; the general driver should satisfy any of $L 1, L 2$ or $L 3$; and the risk-based driver can change lanes in any of $L 1, L 2, L 3$ or $L 4$. The lane-changing rules corresponding to the state of adjacent vehicle are presented as a property (i.e., neighbor-pos) in the WM. The value of neighbor-pos is an 8-bit $0-1$ component. When any state from $L 1$ to $L 4$ and from $R 1$ to $R 4$ is satisfied, the value of the corresponding bit is 1 ; otherwise, it is 0 .

\subsection{Traffic Congestion Situation within Sight Distance of Guidance Information on VMS}

Traffic congestion within sight distance of the VMS is one of the bases for choosing downstream roads in the traffic guidance and compliance scenario. Road occupation in the field of vision is adopted to represent congestion. The degree of congestion at time $t$ is denoted by $p(t)$ :

$$
p(t)=\frac{\sum_{l=1}^{N_{l}} \sum_{c=1}^{N_{s}(l)} \omega(l, c) h(l, c)}{\sum_{l=1}^{N_{l}} N_{s}(l)},
$$

where: $N_{l}$ represents the lane number; $N_{s}(l)$ is the amount of cells within the visual range of lane $l ; \omega(l, c)$ denotes the weight of cell $c$ in line $l$ to the congestion perception of the driver. A larger $c$ has a smaller $\omega(l, c)$; $h(l, c)$ is a $0-1$ variable, where 1 represents the $c$ th square in lane $l$ is occupied, whereas 0 indicates unoccupied square. The degree of congestion within the sight of the VMS is represented as sense-density, which is an attribute in the WM.

A three-digit variable with three levels is adopted to describe the congestion of the downstream roads shown on the VMS. The color on the VMS shows the degree of congestion: $\mathrm{R}$ (red) indicates severe congestion, $\mathrm{Y}$ (yellow) general congestion, and G (green) free-flowing traffic. VMS-sign in the WM shows the information on the VMS. Its three components represent the left, forward, and right orderly.

\section{Memory Representations}

\subsection{Working Memory}

WM is the space where the short-term memory unit is stored to reflect knowledge related to the current situation, such as the current state and operator. WM consists of many objects that contain a set of attributes and values, represented by a hierarchical structure. Fig. 5 shows that the initial state of the agent is $S 1$ in the guidance and compliance scenario. The sub state of $S 1$ is $S 0$. The agent runs in Lane 2 with its destination being Zone 3 under $S 1 ; I 1$ is the input and output of $S 1$ through the input-link and output-link attributes, respectively. The identifier of input-link is $I 3$. The only attribute of $I 3$ is a road denoted by $R 1$, which represents the information perceived (related to the road) from the external environment. The three attributes of $R 1$ are VMS-sign, sense-density, and neighbor-pos. VMS-sign represents the congestion in the downstream roads shown on the VMS; sense-density represents the sensed congestion in the current road; and neighbor-pos represents the state of the adjacent vehicle, to satisfy the lane-changing rules in Section 3.3. The identifier of the output-link is $I 2$, which represents all the possible output. The current operator shown in Fig. 5 is changing destination.

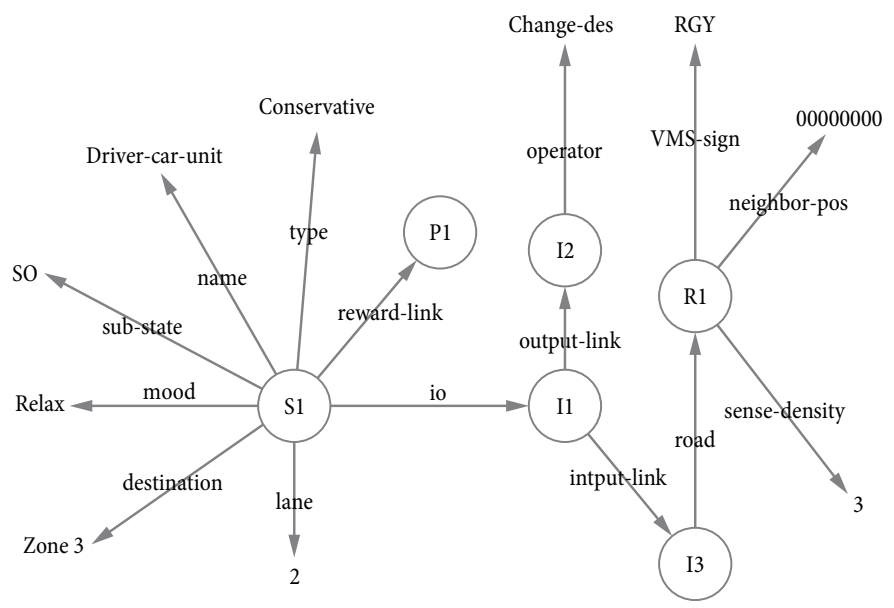

Fig. 5. Hierarchical structure of WM in SOAR 


\subsection{Long-Term Memory}

LTM is the space that stores all the achievements that can be created by users to complete specific functions. The LTM of the agent contains procedural memory and episodic memory. Both memories are represented by productions. Procedural memory represents consolidated knowledge, whereas episodic memory provides cues when the procedural memory exhibits inefficient performance.

The production uses if-then logic to represent condition-action. if represents the conditions governing the actions and then represents individual actions or behaviors. The following equation is an initial rule in LTM, written by stylized and precise representing form.

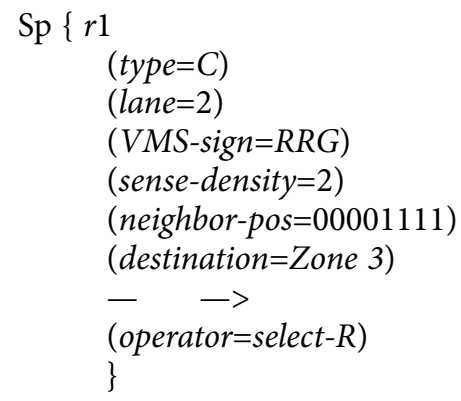

$S p$ stands for 'SOAR production' and starts every rule; ' $\{$ ' starts the body of a rule, whereas ' $\}$ ' ends the rule. The name of the rule immediately follows the ' $\{$ '; $\rightarrow$ separates the if and then parts of the value. The name of the production rule is $r 1$, which means that when the characteristic of the driver is conservative, the signs of the VMS are $R, R$ and $G$; the sensed congestion is severe; the destination is Zone 3; and the conditions required to turn right are satisfied. Hence, the driver selects the road on the right. Only the partial initial rules of the SOAR agent are set, and it will learn to gradually update and add new rules in the process of decision making and feedback. Regardless of how perfect the initial rules are, they cannot contain or correctly contain all the thinking for decision making and preferences. Therefore, a period of training is required to complete the rule base and create realistic preferences, and enable the agent to simulate traffic guidance compliance behaviors better. The effect of learning mechanisms is critical to the training process.

\section{Decision Cycle and Learning Mechanisms}

\subsection{Decision Cycle}

The decision cycle of the SOAR agent involves moving the current state to the goal state by proposing, comparing, selecting, and applying an operator. One rule in the rule base of LTM contains conditions, an operator that matches the conditions in the rule, and the numeric preference of the operator. Four kinds of operators are included in the present study. The first operator selects the direction (including selecting the forward, left or right direction). The second operator changes the congestion situation; according to the external environment and experience, the drivers infer the downstream road conditions that they believe will be the most consistent with the truth. The third operator changes the driving goal from saving money (saving time) to saving time (saving money); saving money requires the drivers to choose the shortest path to the destination, and saving time requires the drivers to choose the path that entails the shortest travel time. The fourth operator changes the mood of the drivers; the drivers may feel relaxed when the accuracy of matching is high, whereas they may become impatient when accuracy is low. The initial state is transferred to the goal state through many intermediate states by the operators. The decision cycle of the SOAR agent is shown in Fig. 6.

During input, the elements of the WM are created via perception and then valued. During elaboration, the contents of perceived information in the WM are matched against the if parts of the rules in the LTM. During operator proposal, all the matched rules are triggered, and the operators in the triggered rules and their preferences are generated. During operator selection, the best operator of the current state is selected among the proposed operators by their preferences. If knowledge cannot sufficiently support decision making, such as when rules cannot be matched or the proposed operators cannot be compared, an impasse occurs and chunking begins. The sub states and the episodic memory are then used to support decision making. During operator application, when the operator selects a direction, then action outputs directly, whereas the other operators (changing the congestion situation, the driving goal, or moods) are selected, then the partial components of the current state are changed to an intermediate state by the operator. In turn, the intermediate state becomes the current state, and persists in the next decision-making cycle to move the problem to the goal state.

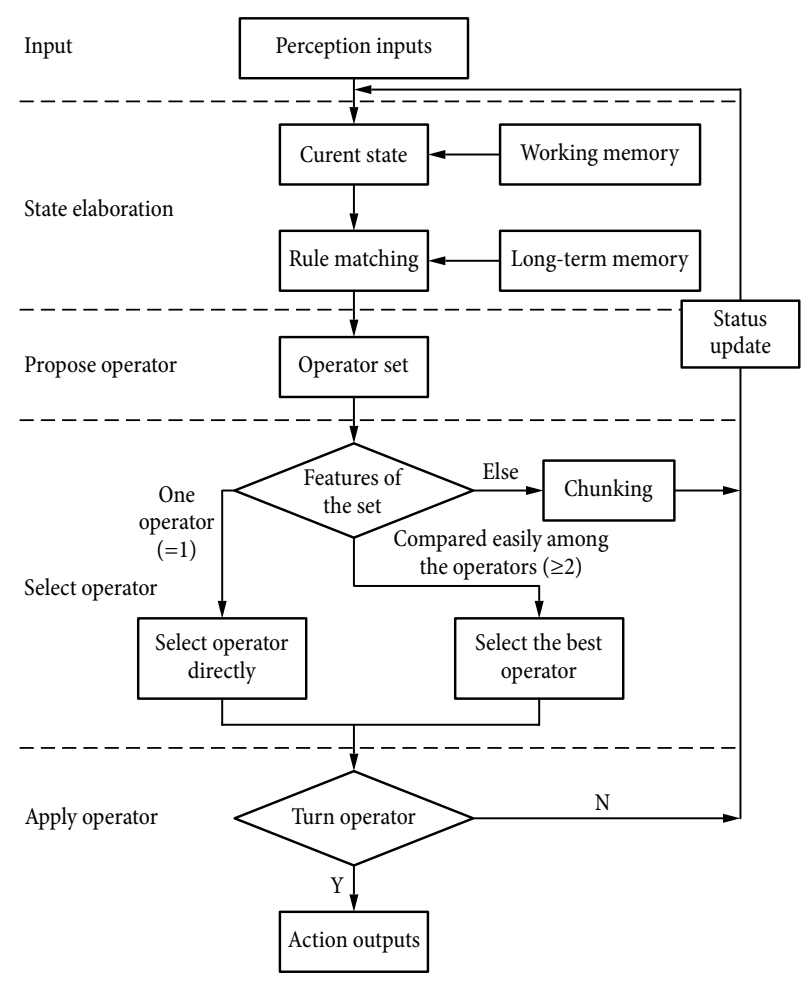

Fig. 6. Decision-making cycle 


\subsection{Learning Mechanisms}

In the present study, the learning mechanisms of SOAR agent include chunking, episodic learning, and reinforcement learning. The procedural memory and episodic memory are updated by chunking and episodic learning, respectively. Regardless of what kinds of memory are used to complete the decision-making process, reinforcement learning is necessary for updating preferences.

\subsubsection{Chunking}

Chunking is a basic learning mechanism for expanding the rules of the SOAR agent. When the rule system is unable to support the agent to make decisions, the current state of the agent is called an impasse. An impasse signifies that the current WM has no available operators to move through the problem space; thus, a new rule is automatically created to solve the impasse. The productions and episodic memories which are related to the achieved result in the LTM are analyzed to establish the chunking rules.

Chunking consists of three parts: the conditions of an impasse, the methods for solving it, and the formation of a chunking rule. The characteristics of the operator set are the basis for operator selection (Fig. 6). If the operator set is null or the difference between the best operator and the second best operator is smaller than $\tau(s)$ which is the threshold value of selecting the operator directly corresponding to the current state $s$, then an impasse occurs. The procedure for solving an impasse is as follows: first, the attributes of the sub state are searched, and the triggered rules of the sub state are used to move the current state (the super state of the sub state) to the next. If an impasse also occurs in the sub state or its attribute is nil, the episodic memories related to the current state $s$ are searched to select the best operator. If there are no cues relating to $s$ in the episodic memories, then the match accuracy decreases by a step size of $\lambda$. Subsequently, the current matched operators in the LTM are searched to move to the goal state. A chunking operator is added into the state in which the impasse occurs if the same chunking rule is matched $\eta_{g}$ consecutive times and the driving time of each is within the expectation which is:

$$
T\left(s_{e}\right)-T_{e}\left(s_{e}\right) \leq \eta_{z},
$$

where: $\eta_{z}$ is the threshold of updating rules; $T\left(s_{e}\right)$ represents the real driving time with goal state $s_{e} ; T_{e}\left(s_{e}\right)$ represents the expected driving time with $s_{e}$;

$$
T_{e}\left(s_{e}\right)=T_{d}(t, l)+T_{v}[v(l)],
$$

where: $T_{d}(t, l)$ is the mean driving time on road $l$ at time $t$, representing the experience of the driver; $T_{v}[v(l)]=\bar{T}(v, l)-T_{r}(l)$ denotes the effect of the congestion shown by guidance information on driving time; $\bar{T}(v, l)$ denotes the mean driving time on road $l$ when the guidance information is $v ; T_{r}(l)$ represents the reference driving time on road $l$. In the present study, $T_{r}(l)$ is the real driving time when the road occupancy rate is 0.5 .

\subsubsection{Episodic Learning}

The rule cues, which have not become procedural rules, are stored as episodic memory. The episodic memory is used by the SOAR agent to simulate the scene memory function of the drivers. When the procedural memory is incapable of supporting a decision, the episodic memory is tapped. The rule cues entering into episodic memory include the following categories:

- when $T\left(s_{e}\right)-T_{e}\left(s_{e}\right) \leq \eta_{z}$, the chunking rules are temporarily stored in the episodic memory. If the same chunking rule is generated $\eta_{g}$ consecutive times, then the rule is transferred to the procedural memory;

- when the utilization ratio of procedural rules denoted by $p_{c}$ in the procedural memory are lower than the critical ratio denoted by $P_{C}$ :

$$
p_{c}=\frac{n u_{i}+c_{c}^{1}}{n s_{i} \beta_{c}+c_{c}^{2}},
$$

where: $n u_{i}$ represents the using times of rule $i$ after it is generated; $n s_{i}$ represents the decision times between the current cycle and the cycle that generates rule $i ; c_{c}^{1}, c_{c}^{2}, \beta_{c}$ are all constants.

To simulate the amnesia of the drivers, when the utilization ratio of the episodic memory denoted by $p_{q}$ is lower than the critical low bound denoted by $P_{Q}^{L}$, the memory is deleted:

$$
p_{q}=\frac{n u_{i}+c_{q}^{1}}{n s_{i}^{\beta_{q}}+c_{q}^{2}},
$$

where: $c_{q}^{1}, c_{q}^{2}, \beta_{q}$ are all constants. In addition, when $p_{q}$ is higher than the upper bound denoted by $P_{Q}^{U}$, the corresponding episodic memory is added automatically into the procedural memory.

\subsubsection{Reinforcement Learning}

The source of reinforcement learning is the feedback from the external environment, which can adjust the expectation of future rewards. These rewards are then used to choose the most expected actions in the future. The guidance compliance agent connects driving time to the total feedback of the applied operator. $p_{r}\left(s_{e}\right)$ represents the total feedback when the final state $s_{e}$ is achieved; it is calculated by $p_{r}\left(s_{e}\right)=\left[T\left(s_{e}\right)-T_{e}\left(s_{e}\right)\right]^{\alpha}$, where $\alpha$ is set as 0.5 . As the decision cycle of the traffic guidance compliance agent involves multiple states and operators, the total preference translated from the total feedback should be allocated to all the related operators. The allocation proportion of each operator depends on the distance between its corresponding state and the goal state. The distance between the intermediate state denoted by $s_{k}$ and the goal state denoted by $s_{e}$ is determined by the changed attributes using the following equation:

$$
\begin{aligned}
& d\left(s_{k}, s_{e}\right)=\left(\left|d_{s}\left(s_{e}\right)-d_{s}\left(s_{k}\right)\right|^{f_{1}}+\right. \\
& \left.\left|d_{e}\left(s_{e}\right)-d_{e}\left(s_{k}\right)\right|^{f_{2}}+\left|m_{o}\left(s_{e}\right)-m_{o}\left(s_{k}\right)\right|^{f_{3}}\right)^{f_{4}},
\end{aligned}
$$

where: $d_{s}\left(s_{e}\right), d_{e}\left(s_{e}\right)$ and $m_{o}\left(s_{e}\right)$ represent the sensed congestion degree, the position number of des- 
tination area and the temper level of the driver under $s_{e}$, respectively; $d_{s}\left(s_{k}\right), d_{e}\left(s_{k}\right)$ and $m_{o}\left(s_{k}\right)$ are the same meanings under $s_{k} ; f_{1}, f_{2}, f_{3}, f_{4}$ are all constants; $\lambda\left[d\left(s_{k}, s_{e}\right), r\left(s_{k}\right)\right] p_{r}\left(s_{e}\right)$ is the feedback preference of $o\left(s_{k}\right)$, which corresponds to the $k$ th state in the state transition path of decision cycle $t ; \lambda\left[d\left(s_{k}, s_{e}\right), r\left(s_{k}\right)\right]$ is the weighing of $p_{r}\left(s_{e}\right)$ for allocation to $o\left(s_{k}\right)$. Moreover, it is the function of $d\left(s_{k}, s_{e}\right)$ and $r\left(s_{k}\right)$ which is the transition path that involves $s_{k}$.

$$
\lambda\left[d\left(s_{k}, s_{e}\right), r\left(s_{k}\right)\right]=\frac{\frac{1}{d\left(s_{k}, s_{e}\right)}}{\sum_{i=1}^{r\left(s_{k}\right) \mid} \frac{1}{d\left(s_{i}, s_{e}\right)}},
$$

where: $\left|r\left(s_{k}\right)\right|$ is the number of states involved in $r\left(s_{k}\right)$.

\section{Numerical Simulation}

\subsection{Simulation Environment}

The road network shown in Fig. 7 is adopted to simulate the traffic guidance compliance behaviors of agents. The road network consists of five sections $(s 1-s 5)$ and four nodes (1-4). $s 1$ is a three-lane road, which corresponds to left-turning, forward-going, and right-turning, whereas the other sections only have one lane; the lengths of the roads are shown in Fig. 7. The VMS is set on $s 1, d \mathrm{~m}$ away from the Node 2. Real-time traffic congestion is released as the guidance information and shown on VMS every five minutes. The different colors stand for different road occupancies: green is for $[0,0.5]$, yellow for $(0.5,0.7]$ and red for $(0.7,1.0]$. Node 2 uses two-phase control signals with a fixed period of $90 \mathrm{~s}$, whereas the other nodes have no control signals. A microscopic traffic flow simulation platform based on cellular automata is adopted; the length of the cell is $7.5 \mathrm{~m}$, and the free flow speed is $60 \mathrm{~km} / \mathrm{h}$. The vehicles are required to pass along the VMS repeatedly to study the effects of VMS locations and change law of drivers' guidance compliance behaviors during a long period after adding a new VMS on $s 1$. Hence, the vehicles enter $s 1$ again through Node 1 if the departure frequency of Node 1 is matched when the vehicles exit Node 4. The departure frequency of Node 1 ranges from 0.2 to 0.8 with cyclical change to simulate peak and off-peak hours. The departure frequency of Node 1 is $(0.6+\delta)$ units/sec during peak hours and $(0.4+\delta)$ units/sec during off-peak hours; $\delta$ is a random number between $[-0.2,0.2]$. The total simulation time is $150 \mathrm{~h}$; peak hours are alternated with offpeak hours every hour.

\subsection{Simulation Results and Analysis}

\subsubsection{Effects of VMS Locations on Traffic Flow}

In the range of 0 to $200 \mathrm{~m}$ ahead of Node 2, VMS is located in different locations on $s 1$ with intervals of each $20 \mathrm{~m}$. 11 simulations are conducted under the simulation conditions depicted in Section 6.1. Fig. 8 shows the average travel time of drivers during peak and off-peak hours with VMS in different locations.

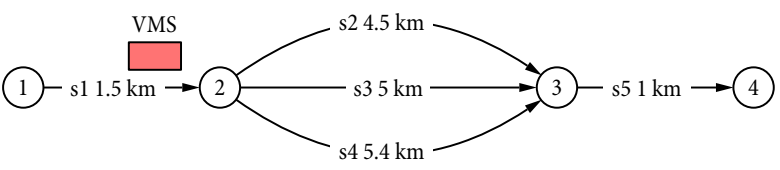

Fig. 7. Simulation road network

Fig. 8 shows that the location of the VMS with the least average travel time is $100 \mathrm{~m}$ away from Node 2 during off-peak hours $-8.6 \%$ less than the time when the VMS is located at the intersection. The effect of the VMS locations on travel time is greater during peak hours. The average travel time is larger than $900 \mathrm{~s}$ when the VMS is located near Node 2, whereas it is only $760 \mathrm{~s}$ when the VMS is $140 \mathrm{~m}$ away from Node 2, reducing travel time by $16.7 \%$. Clearly, the location of a VMS is important in keeping roads smooth. Moreover, the optimum locations of VMS during peak and off-peak hours are different. During peak hours, the VMS should be located much farther from the downstream intersection. During peak hours, the traffic flow is heavy and the headway time is smaller, especially near the downstream intersection. If the VMS is too close to the intersection, the adjacent vehicle is not allowed to change lanes even though the driver wanted to do so. Meanwhile, the distance between the VMS and the downstream intersection should not be as far as possible. When the VMS is too far from the downstream roads, the drivers will misjudge the conditions of downstream roads and choose the wrong route, thus affecting the average travel time of groups. Based on the simulation results, setting the VMS 100 to $140 \mathrm{~m}$ away from Node 2 is suitable.

Fig. 9 shows the average speeds of the vehicles on the downstream roads of VMS ( $s 2, s 3$ and $s 4$ ) in the last $6 \mathrm{~h}$ when the VMS is set at Node 2 and $140 \mathrm{~m}$ away from the front of Node 2. The traffic flow presents evident cyclical features during peak and off-peak hours, but VMS locations have different effects on the speed of traffic flow on the downstream roads. When the VMS is set at Node 2, the speeds of traffic flow on roads $s 2$, $s 3$ and $s 4$ during off-peak hours slightly differ, but the difference reaches up to $20 \%$ during peak hours. This is because traffic flow is small during off-peak hours, and each downstream road is relatively smooth; thus,

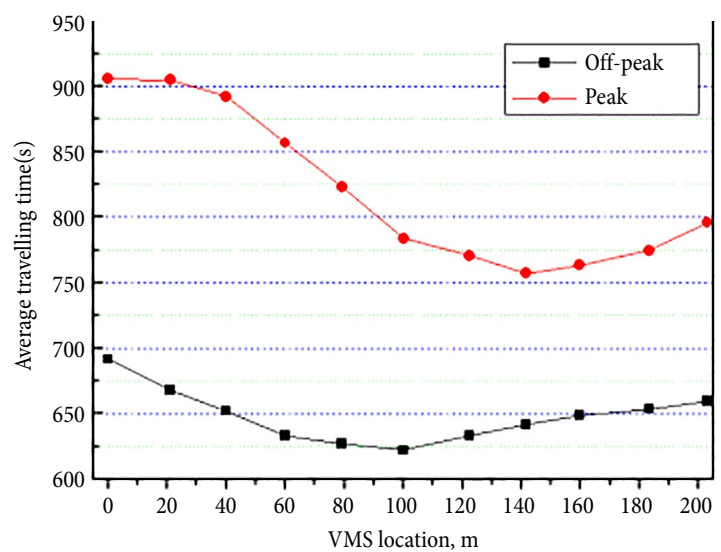

Fig. 8. Average travel time with VMS in different locations 

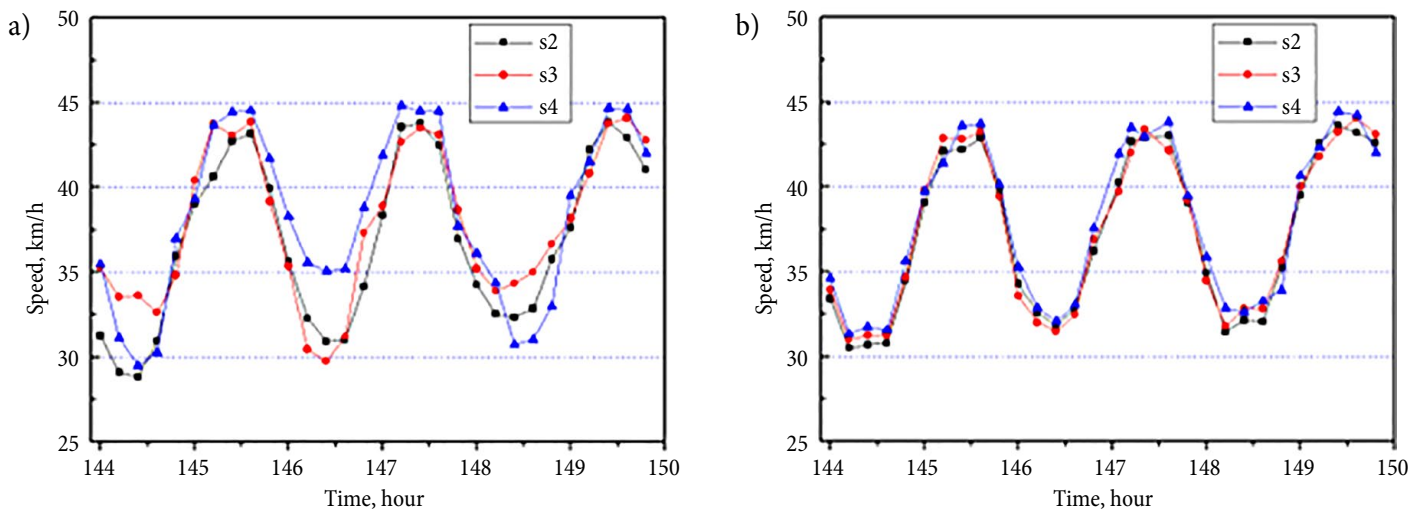

Fig. 9. Average speed of $s 2, s 3$ and $s 4$ in the last $6 \mathrm{~h}$ when the VMS is set at Node 2 (a) and $140 \mathrm{~m}$ from the front of Node 2 (b)

the road capacity is greater than traffic flow, and the drivers can choose any road and travel at high speeds. Therefore, the difference in speed among the vehicles is small. During peak hours, the capacities of the partial downstream roads of the VMS are close to saturation with the increase of traffic flow, whereas the other roads still remain relatively great capacities. In such a case, the drivers choose the different downstream roads of the VMS, which in turn affects their travel time more considerably; hence, the speed of traffic flow on different roads varies obviously. Fig. 9a shows that when the VMS is set near Node 2, the traffic flow along the VMS cannot be effectively allocated to the downstream roads, which results in the unbalanced distribution of traffic flow on the downstream roads. Fig. $9 \mathrm{~b}$ shows that when the VMS is set $140 \mathrm{~m}$ away from the downstream roads, the average travel speeds on the downstream roads are consistent regardless of peak and off-peak hour. The traffic flow on the downstream roads is equally distributed, indicating that the effect of the guidance effect is better when the VMS is set a bit further from the intersection.

\subsubsection{Lane-Changing Times}

Once a vehicle enters within sight distance of the VMS, its lane-changing behavior is an important indicator that represents drivers' guidance compliance. Fig. 10 shows

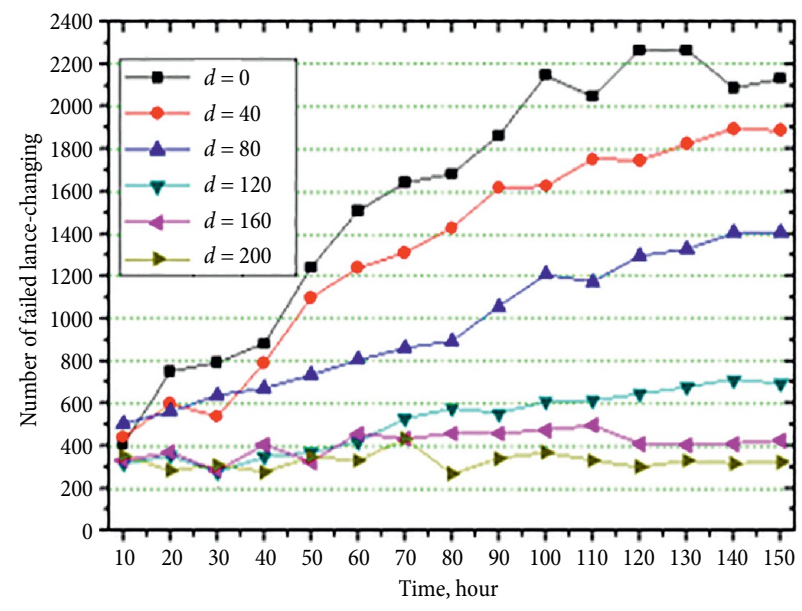

Fig. 10. Numbers of failed lane-changing with VMS in different locations the statistical failed lane-changing times within the length of one cell every $10 \mathrm{~h}$ when VMS is positioned at different locations (i.e., $0,40,80,120,160$, and $200 \mathrm{~m}$ away from the intersections), reflecting the influence of VMS location on the traffic guidance compliance rate of drivers.

All the failed lane-changing numbers are not large in the initial stage of the simulation. As simulation progresses, the difference among the drivers' failed lanechanging times, which is affected by VMS locations, increases gradually. When VMS is closer to the intersection, drivers fail to change lanes more frequently. Hence, the different locations of the VMS have a huge effect on the consistence of drivers' guidance compliance will and their guidance compliance behaviors. When the location of the VMS is closer to the intersection, drivers can forecast traffic congestion on downstream roads more accurately once they enter within sight distance of the VMS. Moreover, they can choose the smoothest downstream road more easily, but the probability of failing to change lanes is larger as the traffic flow becomes more congested. Hence, drivers cannot change lanes even if they want to, and the real compliance rate decreases. Therefore, setting the VMS a bit further from the intersection is more effective in increasing the traffic guidance compliance rate of drivers. Nevertheless, the location of the VMS should not be set as far as possible from the intersection. Otherwise, the drivers may inaccurately assess actual traffic congestion on the downstream roads. This, in turn, causes them to doubt the accuracy of the information shown on the VMS. Through multiple processes of feedback and learning, they decrease their will to comply with, even if no other factors (e.g., traffic jams) have an effect on their lane-changing behavior after they have entered within sight distance of the VMS. Their compliance rate drops as well.

\subsubsection{Study Laws of Drivers' Guidance Compliance Behaviors}

Fig. 11 shows the probability of route switching with the same guidance information shown on the VMS and the same traffic condition, but at different hours of the day (i.e., peak and off-peak hours) and different VMS locations (i.e., the distances between VMS and the in- 


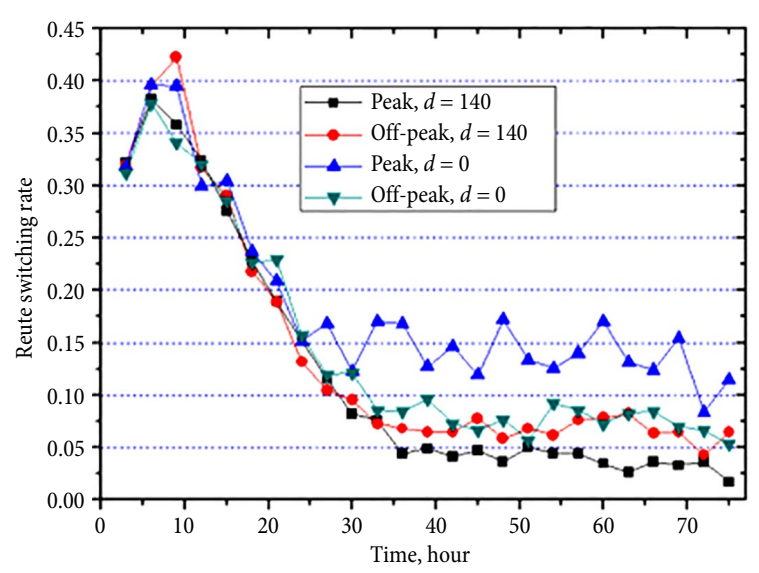

Fig. 11. Route switching rate when the VMS is set at Node 2 and $140 \mathrm{~m}$ from the front of Node 2 during peak and off-peak periods

tersection are 0 and $140 \mathrm{~m}$ ). The simulation is used to study the guidance effects of newly added VMS on $s 1$ and the change laws of these effects. During the initial stage of the simulation, as all the agents are newly added, a certain period is needed for the drivers to experience guidance from the VMS. This stabilizes the cognition of the accuracy of guidance information. Fig. 11 shows that the initial route switching rate is approximately 0.45 , indicating that $45 \%$ of the drivers choose a different route during this period when they encounter the same guidance information twice. As the simulation progresses, the average route switching rate decreases to a steady state around the 30th day. This is the process by which the drivers gradually become familiar with the guidance performance of the VMS. When the drivers adapt to the guidance function of the VMS, the traffic guidance compliance behaviors of groups, which is represented by the guidance compliance rate, stabilizes whether the individual driver obeys the guidance information or not.

Although the average guidance compliance rate of drivers remains stable, the effects of different hours of the day and locations of the VMS on guidance compliance behaviors of drivers are inconsistent. Fig. 11 shows that when the VMS is set $140 \mathrm{~m}$ away from the front of the intersection during peak hours, the route switching rate becomes smaller after the guidance compliance rate stabilizes. The average switching rate is only about 0.05 , indicating that drivers seldom change their guidance compliance behaviors and habits once they have adapted to the guidance function of the VMS during peak hours. The average switching rate also shows that drivers are able to change lanes with their wills. Meanwhile, when the VMS is located $140 \mathrm{~m}$ away from the front of the intersection during off-peak hours, the route switching rate increases slightly (i.e., 0.08 on average). In such a situation, the cost incurred through lane-changing is slight because of the small traffic flow during off-peak hours, and drivers select the downstream roads with certain arbitrariness. Compared with the other three cases, setting the VMS at the intersection during peak hours has the greatest influence on route switching rate.
The average switching rate reaches up to 0.15 , but also fluctuates because when the VMS is located near the intersection. Once the drivers enter within sight distance of the VMS and wish to choose a downstream road, the probability of failing to change lanes becomes huge. The drivers who fail to change lanes are forced into the road corresponding to current lane, resulting in different route choice results under the same conditions.

\section{Conclusions}

In the present study, the SOAR cognitive architecture is adopted to study the formation mechanism and change laws of the guidance compliance behaviors of drivers. Moreover, the effect and reason of setting VMS in different locations are analyzed. On the basis of the simulation results, a range for the optimal VMS location is recommended. The present study is a basic research on the effective implementation of traffic guidance compliance systems, and may provide a theoretical basis to set the optimal VMS location.

The location of a VMS is influenced by many factors, such as information release mode, road environment, vehicle composition and traffic flow etc. The combined influence of these factors is difficult to assess by using a fixed analytical formula. Whereas multi-agent simulation based on the SOAR cognitive architecture is a good platform for integrating these factors, and serves as a comprehensive approach to study complex traffic behavior. The present study describes the perception, memory, decision making and learning which are involved in traffic guidance compliance in detail from the perspective of individual drivers. Moreover, we investigate the effect of VMS locations on the traffic guidance compliance behaviors through group phenomenon from multi-agent simulations. The present study is an attempt to combine cognitive science and simulation methods in studying traffic guidance compliance behaviors. As the research on traffic guidance systems becomes more extensive, and more attention is paid to traffic guidance compliance behaviors, better traffic guidance systems will be developed.

\section{Acknowledgements}

The research described in this paper was substantially supported by grants (50908155, 70971094 and 71371134 ) from the National Natural Science Foundation of China.

\section{References}

Bekhor, S.; Prashker, J. N. 2008. GEV-based destination choice models that account for unobserved similarities among alternatives, Transportation Research Part B: Methodological 42(3): 243-262. http://dx.doi.org/10.1016/j.trb.2007.08.003

Bonsall, P. W.; Joint, M. 1991. Driver compliance with route guidance advice: the evidence and its implications, in $\mathrm{Ve}$ hicle Navigation \& Information Systems Conference Proceedings, 20-23 October, 1991, Dearborn, Michigan, US, Part 2, 47-59. http://dx.doi.org/10.1109/VNIS.1991.205747 
Chowdhury, D.; Wolf, D. E.; Schreckenberg, M. 1997. Particle hopping models for two-lane traffic with two kinds of vehicles: effects of lane-changing rules, Physica A: Statistical Mechanics and its Applications 235(3-4): 417-439. http://dx.doi.org/10.1016/S0378-4371(96)00314-7

Cummings, M. 1994. Electronic sign strategies and their benefits, in Proceedings of the Seventh International Conference on Road Traffic Monitoring and Control, London, UK, 2628 April, 1994, 141-144.

http://dx.doi.org/10.1049/cp:19940443

Daganzo, C. F.; Sheffi, Y. 1977. On stochastic models of traffic assignment, Transportation Science 11(3): 253-274. http://dx.doi.org/10.1287/trsc.11.3.253

Deflorio, F. P. 2003. Evaluation of a reactive dynamic route guidance strategy, Transportation Research Part C: Emerging Technologies 11(5): 375-388. http://dx.doi.org/10.1016/S0968-090X(03)00031-7

Erke, A.; Sagberg, F.; Hagman, R. 2007. Effects of route guidance variable message signs (VMS) on driver behaviour, Transportation Research Part F: Traffic Psychology and Behaviour 10(6): 447-457. http://dx.doi.org/10.1016/j.trf.2007.03.003

Jou, R.-C.; Lam, S.-H.; Liu, Y.-H.; Chen K.-H. 2005. Route switching behavior on freeways with the provision of different types of real-time traffic information, Transportation Research Part A: Policy and Practice 39(5): 445-461. http://dx.doi.org/10.1016/j.tra.2005.02.004

Laird, J. E.; Newell, A.; Rosenbloom, P. S. 1987. SOAR: An architecture for general intelligence, Artificial Intelligence 33(1): 1-64. http://dx.doi.org/10.1016/0004-3702(87)90050-6

Mahmassani, H. S.; Liu, Y.-H. 1999. Dynamics of commuting decision behaviour under advanced traveller information systems, Transportation Research Part C: Emerging Technologies 7(2-3): 91-107.

http://dx.doi.org/10.1016/S0968-090X(99)00014-5

Marinier, R. P.; Laird, J. E.; Lewis, R. L. 2009. A computational unification of cognitive behavior and emotion, Cognitive Systems Research 10(1): 48-69.

http://dx.doi.org/10.1016/j.cogsys.2008.03.004

Rosenbloom, P. S.; Laird, J. E.; Newell, A. 1987. Knowledgelevel learning in Soar, in AAAI-87 Proceedings, 13-17 July, 1987, Seattle, Washington, 499-504.

Swann, J.; Routeledge, I. W.; Parker, J.; Tarry, S. 1995. Results of practical applications of variable message signs (VMS): A64/A1 accident reduction scheme and forth estuary driver information and control system (FEDICS), in Traffic Management and Road Safety. Proceedings of Seminar G Held at the 23rd PTRC European Transport Forum, 11-15 September, 1995, University of Warwick, England, 149-167.

Tarry, S.; Graham, A. 1995. The role of evaluation in ATT development: 4. Evaluation of ATT systems, Traffic Engineering \& Control 36(12): 688-695.

Thakuriah, P.; Sen, A. 1996. Quality of information given by advanced traveler information systems, Transportation Research Part C: Emerging Technologies 4(5): 249-266. http://dx.doi.org/10.1016/S0968-090X(97)82900-2

Wang, Y.; Papageorgiou, M.; Sarros, G.; Knibbe, W. J. 2006. Real-time route guidance for large-scale express ring-roads, in Proceedings of the ITSC'06 IEEE Intelligent Transportation Systems Conference, 17-20 September, 2006, Toronto, Canada, 224-229. http://dx.doi.org/10.1109/ITSC.2006.1706746
Wardman, M.; Bonsall, P. W.; Shires, J. D. 1997. Driver response to variable message signs: a stated preference investigation, Transportation Research Part C: Emerging Technologies 5(6): 389-405. http://dx.doi.org/10.1016/S0968-090X(98)00004-7

Watling, D. 2006. User equilibrium traffic network assignment with stochastic travel times and late arrival penalty, European Journal of Operational Research 175(3): 1539-1556. http://dx.doi.org/10.1016/j.ejor.2005.02.039

Yin, Y.; Yang, H. 2003. Simultaneous determination of the equilibrium market penetration and compliance rate of advanced traveler information systems, Transportation Research Part A: Policy and Practice 37(2): 165-181. http://dx.doi.org/10.1016/S0965-8564(02)00011-3 